Research Report

\title{
Microarray-Based Method for Combinatorial Library Sequence Mapping and Characterization
}

\author{
V. Abécassis, L. Jaffrelo, \\ D. Rickman, L. Aggerbeck, \\ C. Herbert, G. Truan, and \\ D. Pompon
}

Centre National de la Recherche Scientifique, UPR 2167, Gifsur-Yvette, France

\section{INTRODUCTION}

On-chip sequence mapping technology can involve two inverse formats (1-3). In the first one, sequence-specific and radioactively labeled oligonucleotide probes are sequentially hybridized to target DNA bound on filters (4-8). Alternatively, the differential stability of duplexes between DNA being probed and perfectly matched or mismatched oligonucleotide targets bound to a chip can be used for sequence determination (9-12). The second strategy is well adapted for the characterization of a large number of sequence positions on a limited number of DNA to probe, but not for the characterization of a large library of sequences on a limited number of positions, which is better achieved by the first approach. Sequencing by hybridization using short oligonucleotides was used in a large range of applications such as complete DNA sample sequence determination (1), genetic polymorphism analysis (13), overlapping clone ordering in a large library (4), and differentially regulated gene isolation and quantification (7).

Combinatorial libraries of DNA sequences are central to directed evolution approaches of protein engineering (14-16). However, DNA shuffling of sequence variants or gene families $(17,18)$ generally suffers from an inhomogeneous repartition of recombination events $(19,20)$, particularly when the sequence similarity between the parental sequences is low $(21,22)$. These biases dramatically reduce generated diversity and complicate functional screening. Sequence characterization of combinatorial libraries is of interest for evaluating library qualities before expensive functional screening and for allowing their improvement $(19,20)$. Particularly, a large biased library can be converted to a smaller and nonredundant one $(23,24)$ by robotic sorting. Additionally, a change in the sequence signature of a library in response to functional selection is a potentially powerful tool for structurefunction analysis (20).

An approach on macroarrays was recently described for the analysis of small combinatorial libraries in terms of mosaic structure repartition and recombination bias analysis (20). This involved the mapping of libraries of mosaic genes resulting from family shuffling using sequential hybridization of parent-specific radiolabeled probes on filter-bound plasmid DNA. However, important fluctuations in hybridization signal intensities were observed between spots with this single-label technique, which makes interpretation difficult. Such one-probe-ata-time approaches are very sensitive to hybridization and washing conditions and require replicate filters and manual checking of signals for reliability (25). We thus decided to shift to glass-supported microarrays (25-27), which allow higher densities of spots than filters (28), and double-fluorescent labeling techniques $(26,27,29,30)$ that may be less prone to signal interpretation errors. The present work was dedicated to the analysis of the main parameters that control hybridization and sequence discrimination in such an oligonucleotide probe competitive hybridization approach. The application of the method to the characterization of combinatorial libraries is also described. 


\section{MATERIALS AND METHODS}

\section{Plasmids and Libraries}

p1A1/V60 and p1A2/V60 have been described previously $(31,32)$. The three libraries, A, B, and C, were constructed after a PCR-based reassembly of DNase I-fragmented $\mathrm{p} 1 \mathrm{~A} 1 / \mathrm{V} 60$ and $\mathrm{p} 1 \mathrm{~A} 2 / \mathrm{V} 60$ (20). After in vivo recombination in yeast (20), DNA extracted from approximately 5000 clones was transformed twice in Escherichia coli, giving libraries A and B. For library C, the same mixture of PCR-reassembled fragments as the one used for libraries $A$ and $B$ was amplified and inserted in $\mathrm{pCR}^{\circledR} 2.1$ TOPO $^{\circledR}$ using the TOPO-TA Cloning ${ }^{\circledR}$ kit (Stratagene, La Jolla, CA, USA). Three different ligation steps were required to constitute a population of approximately 400 clones containing the cDNA insert (evaluated by PCR amplification directly on $E$. coli colonies). Plasmidic DNA from the pooled clones was prepared and restricted by HindIII digestion, and plasmids containing cDNA inserts were purified by agarose gel electrophoresis, circularized by ligation, and amplified in E. coli, giving library $\mathrm{C}$.

\section{DNA Amplification Procedures}

E. coli DH5-1 strain was transformed by the DNA libraries. Three hundred eighty-four colonies from each library were grown for $24 \mathrm{~h}$ in a 384well plate containing $40 \mu \mathrm{L} \mathrm{LB}$ medium supplemented with $100 \mu \mathrm{g} / \mu \mathrm{L}$ ampicillin. One microliter of each microculture was used to amplify the cytochrome P450 ORFs as described previously (20). The PCR program was $94^{\circ} \mathrm{C}$ for $3 \mathrm{~min}$; 30 cycles of $1 \mathrm{~min}$ at $94^{\circ} \mathrm{C}, 1 \mathrm{~min}$ at $56^{\circ} \mathrm{C}$, and $3 \mathrm{~min}$ at $72^{\circ} \mathrm{C}$; and finally a 7 -min step at $72^{\circ} \mathrm{C}$. Bacterial clones previously transformed by $\mathrm{p} 1 \mathrm{~A} 1 / \mathrm{V} 60$, p1A2/V60, or pYeDP60 were also used for PCR amplifications. Amplified DNAs were purified with the Multiscreen ${ }^{\mathrm{TM}}$ 384-well filter plate (Millipore, Bedford, MA, USA) using the manufacturer's recommendations, and DNA concentrations were measured with a PowerWaveX ${ }^{\mathrm{TM}}$ Microplate Spectrophotometer (BioTek Instruments, Winooski, VT, USA). After drying, each DNA sample was re- suspended in $8 \mu \mathrm{L}$ spotting solution, resulting in concentrations greater than $100 \mathrm{ng} / \mu \mathrm{L}$ for $95 \%$ of the wells (33).

\section{Slide Preparation and Spotting}

Poly-L-lysine (Labo Moderne, Paris, France) and amino-silane (Sigma, St. Louis, MO, USA)-coated slides were used for spotting with a GeneTAC ${ }^{\circledR} \mathrm{G} 3$ robot (Genomic Solutions, Ann Arbor, MI, USA) with $200-\mu \mathrm{m}$ pins. DNA samples were printed in quadruplicate with a center-to-center spacing of 300 $\mu \mathrm{m}$. After spotting, the slides were dried at room temperature, and the DNA was UV-cross-linked for $5 \mathrm{~min}$ at $250,000 \mu \mathrm{J} / \mathrm{cm}^{2}$. Just before use, the slides were placed for $5 \mathrm{~min}$ in $2 x$ $\mathrm{SSC} / 0.1 \% \mathrm{SDS}, 3 \mathrm{~min}$ in $0.2 \times \mathrm{SSC}$ (34), and $2 \mathrm{~min}$ in double-distilled water and then dried in a SpeedVac ${ }^{\circledR}$ (Thermo Savant, Holbrook, NY, USA) for $5 \mathrm{~min}$. One slide of each series was placed in a 1:10,000 dilution in water of SYBR ${ }^{\circledR}$ Green II solution (Molecular Probes, Eugene, OR, USA) for 5 min, followed by multiple washes in double-distilled water before scanning at $532 \mathrm{~nm}\left(\mathrm{GenePix}^{\circledR}{ }^{4}\right.$ 4000B; Axon Instruments, Union City, CA, USA).

\section{Probe Choices and Hybridization Procedures}

Six oligonucleotide probe pairs (ESGS, Cybergene, Evry) were designed as previously reported (Table 1). Two probes from a single set target the same part of the sequence and were used together for slide hybridization. All steps were performed in an automated hybridization station (Genomic Solutions). Slides underwent a 30 -min prehybridization step at $42^{\circ} \mathrm{C}$ in $5 \times$ SSC, $0.2 \%$ SDS; $5 \times$ Denhardt's reagent (34). The same solution was used for hybridization. Four parameters were evaluated using probe set 2: probe quantity, hybridization duration, hybridization temperature, and washing temperature. For each hybridization, three washing steps, of two times $30 \mathrm{~s}$, were performed. The first washing step was done with $2 \times \mathrm{SSC} / 0.2 \% \mathrm{SDS}$, the second with $0.2 \times \mathrm{SSC}$, and the third with $0.05 \times$ SSC. Only the temperature of the second washing step was varied; the other two washing steps were done at $25^{\circ} \mathrm{C}$. Each probe set was used to hybridize different slides.

\section{Data Processing}

The hybridization data were processed using homemade software (see accompanying paper) that allowed subpopulation filtering and automated sequence attribution. The spotter information file and the slide fluorescence data were first associated to allow spot identification. Medians of the fluorescence intensities of pixels from each spot minus the medians of the local background were calculated giving $\mathrm{FC}_{635}$ (red) and $\mathrm{FC}_{532}$ (green) values, respectively. A discrimination index R2 for each spot was calculated as follows:

$$
\begin{aligned}
& \mathrm{R} 2=3-\frac{4}{\Pi} \times A t n\left(\frac{-\mathrm{FC}_{635}}{\mathrm{FC}_{532}}\right) \text { if } \mathrm{FC}_{635} \geq 0 \text { and } \mathrm{FC}_{532}<0 \\
& \mathrm{R} 2=-3-\frac{4}{\Pi} \times A t n\left(\frac{-\mathrm{FC}_{635}}{\mathrm{FC}_{532}}\right) \text { if } \mathrm{FC}_{635}<0 \text { and } \mathrm{FC}_{532} \geq 0 \\
& \mathrm{R} 2=\frac{4}{\Pi} \times A t n\left(\frac{\mathrm{FC}_{635}}{\mathrm{FC}_{532}}\right)-1 \text { in all other cases }
\end{aligned}
$$

$\operatorname{Atn}(\mathrm{u})$ is the trigonometric arc-tangent value of $u$ (in radian units) using the function definition suitable for the angular range $-\Pi \mathrm{P} / 2$ to $+\Pi \mathrm{P} / 2$.

Cross-correlation coefficients $\left(\rho_{\mathrm{x}, \mathrm{y}}\right)$ were calculated as follows:

$$
\begin{aligned}
& \rho_{x, y}=\frac{\operatorname{Cov}(X, Y)}{\sigma_{x} \cdot \sigma_{y}} \text { where }-1 \leq \rho_{x, y} \leq 1 \text { and } \\
& \operatorname{Cov}(X, Y)=\frac{1}{n} \sum_{i=1}^{n}\left(x_{i}-\mu_{x}\right)\left(y_{i}-\mu_{y}\right) \\
& \mu_{x}=\frac{1}{n} \sum_{i=1}^{n} x_{i} ; \mu_{y}=\frac{1}{n} \sum_{i=1}^{n} y_{i} ; \\
& \sigma_{x}=\sqrt{\frac{n \sum x^{2}-\left(\sum x\right)^{2}}{n(n-1)}} ; \\
& \sigma_{y}=\sqrt{\frac{n \sum y^{2}-\left(\sum y\right)^{2}}{n(n-1)}}
\end{aligned}
$$

\section{RESULTS}

\section{Design of a Sequence Mapping Strategy Based on DNA \\ Microarrays for Combinatorial Library Analysis}

Mosaic structures were spotted as approximately 1900-bp PCR products covering the ORF and a limited amount of the promoter and the terminator flanking 


\section{Microarray Technologies}

sequences. As described in Table 1, six probe pairs were synthesized to analyze combinatorial libraries spotted on glass slides. Each probe pair was designed using previously described probe positions (20). Their initial length ranged from 19 to 25 . In each pair, only $4-12$ bases matched both sequences. In some cases, oligonucleotides were lengthened from 1 to 4 bases to adjust the $T_{m}$ to around $68^{\circ} \mathrm{C}$. CYP1A1 and $C Y P 1 A 2$ probes were 5'-labeled with $\mathrm{Cy}^{\mathrm{TM}} 3$ and Cy5 dyes, respectively. Examination of commercially available modified glass slides in terms of spot homogeneity, intensity, and background, following competitive hybridization with labeled oligonucleotides, was performed. PolyL-lysine-coated slides and blocking by Denhardt's reagent proved to be a good cost-to-quality compromise over aminosilane slides and more sophisticated blocking strategies (see http://cmgm. stanford.edu/pbrown for protocols).

Probe concentrations ranging from 0.9 to $90 \mathrm{nM}$ were tested, and the 9- to 45-nM range was found to give suitable signal-to-noise ratios. This range of concentration is $10^{3}$ - to $10^{4}$-fold higher than that for a specific cDNA in transcriptome analysis but remains very low with respect to the scale of synthesis for standard oligonucleotides. Figure 1 shows the effects of the modulation of different hybridization parameters. Analysis of hybridizations lasting between 30 min and $15 \mathrm{~h}$ showed that at least $75 \%$ of the maximum signal was reached in $30 \mathrm{~min}$ (Figure 1A). This fast hybridization phase was followed by a slow increase in the signal up to $15 \mathrm{~h}$. A similar behavior was observed for both the Cy3- and the Cy5-labeled probes. The background also slightly increases with hybridization times. Analysis of the time dependence of the ratio of the red and the green signal indicated little changes for most spots and a high reproducibility among replicas (not shown). Nevertheless, a careful examination of the data showed that a very limited number of green spots can progressively turn yellow with increasing times of hybridization. Increasing the hybridization temperature (Figure 1B) from $42^{\circ} \mathrm{C}$ to $58^{\circ} \mathrm{C}$ resulted in a progressive decrease of the fluorescence intensity after hybridization without a clear temperature transition, a somewhat surprising result for short oligonucleotide probes. Similar behaviors were observed for washing temperatures (Figure 1C), with almost a complete loss of the signal compared to the background at $55^{\circ} \mathrm{C}$, a temperature still significantly lower than the evaluated fusion temperature of the oligonucleotides (Table 1). Optimal conditions were found to correspond to a probe concentration of $9 \mathrm{nM}$, a hybridization temperature of $42^{\circ} \mathrm{C}$, and a washing temperature of $35^{\circ} \mathrm{C}$. The system has proven to be tolerant to variations in experimental conditions, allowing the use of the same conditions for all probe sets without further adjustment.

\section{Sequence Assignment Based on a Polar Representation of Fluorescence Signals}

Figure 2 illustrates the analysis of the relationship between $\mathrm{Cy} 3$ and $\mathrm{Cy} 5$ signals following hybridization of slides with probe set number 2 . The $\mathrm{Cy} 3$ and Cy5 signals were found to be highly variable from spot to spot (Figure $2 \mathrm{~A}$ ). However, the signal dispersion was limited (less than 20\%) when intensities of quadruplicated spots corresponding to the same PCR product were compared, suggesting that the signal heterogeneity is mainly sample-specific (even if the samples concentrations were previously adjusted). Indeed, some physical differences in the state of DNA on the chip, which is not related to DNA concentration but more likely to residual impurities, strongly modulated the oligonucleotide probes hybridization efficiencies. Signal fluctuation could be reduced by a tight control of PCR product amplification and purification, which is, however, not practicable on large libraries. Fortunately, the advantage of competitive hybridization targeting overlapping sequences is that the signal of one probe can be used as a kind of "internal reference" for the other probe. A polar transformation leading to the calculation of a discrimination index R2 (see Materials and Methods) was designed to take advantage of this property. The rationale for the choice of the mathematic transfor- 
mation used is developed in an accompanying paper. As illustrated in Figure $2 \mathrm{~B}$, such a transformation gives rise to a clear-cut trimodal frequency for the observed R2 indexes in the library. The principal modes correspond to populations exhibiting CYP1A1 type (-1 centered), $C Y P 1 A 2$ type ( +1 centered), and unexpected sequence ( 0 centered), respectively. This last central and more disperse population is constituted by yellowish spots generally representing less than $5 \%$ of the total population. Se- quencing and restriction analysis indicated that this population is mainly linked to mixtures of sequences or mutated sequences. Depending on the quality of the PCR amplification and on the probe sequence and similarity, the trimodal distribution can turn into more complex distributions that require more complex data processing and particularly the use of specific filters to eliminate nonpertinent subpopulations. Such treatment is described in detail in the accompanying paper (Abécassis et al.,

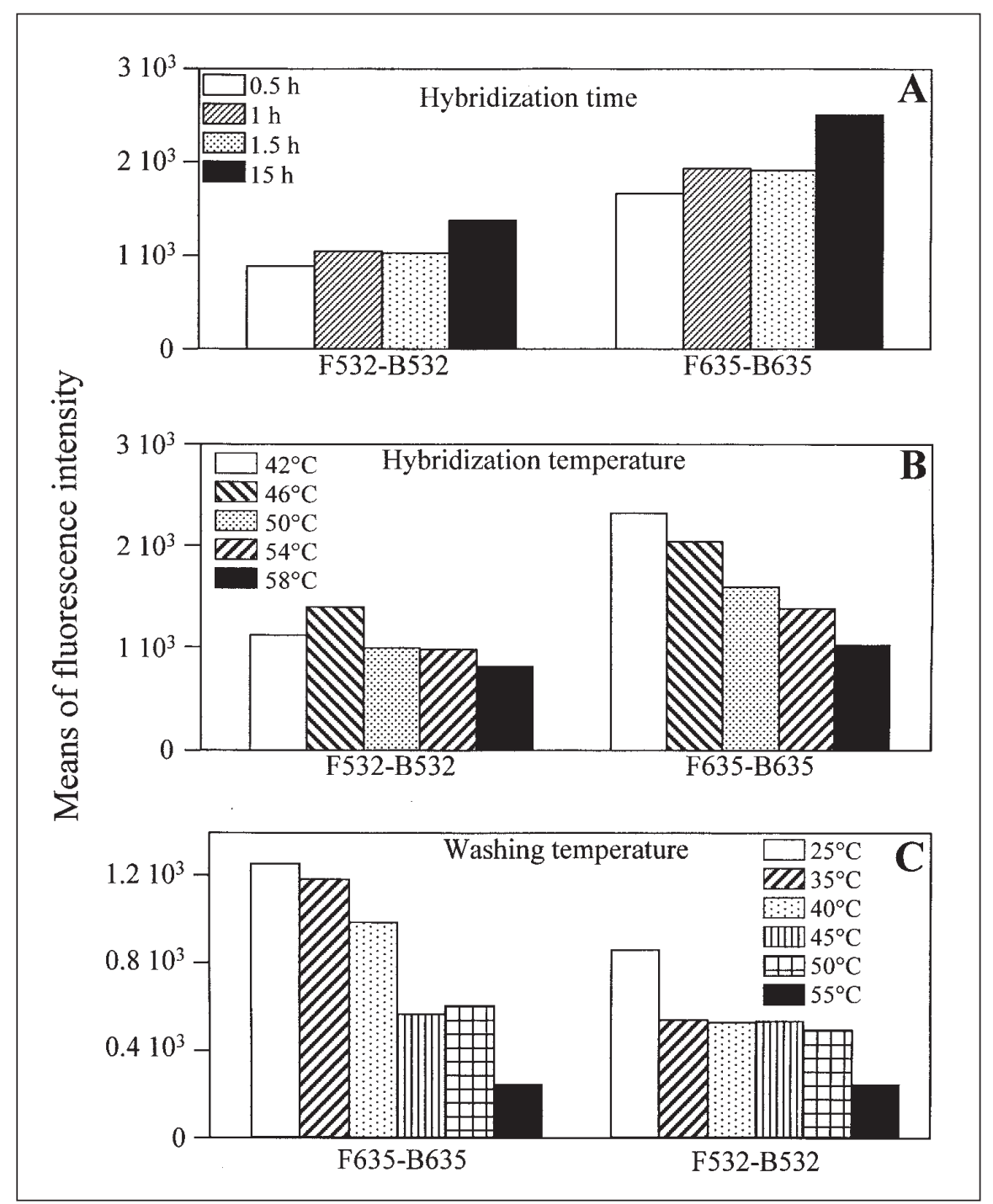

Figure 1. Differential hybridization of a Cy3- and Cy5-labeled oligonucleotide probe pair (probe set 2) on a slide having quadruplicate spotting of the libraries studied. The means of corrected 532and 635-nm intensities [spot raw fluorescence (F) minus local fluorescence background around spot (B)] were calculated for all of the population as a function of hybridization time, hybridization temperature, or washing temperature. (A) Effect of hybridization time. The slides were hybridized in the standard buffer with 1 pmol each probe. (B) Hybridizations at variable temperatures were performed during $1.5 \mathrm{~h}$ using $1 \mathrm{pmol}$ each probe. (C) To test washing temperatures, hybridizations were performed $42^{\circ} \mathrm{C}$ for $1.5 \mathrm{~h}$ with 1 pmol each probe. Other conditions were as described in the Materials and Methods. 


\section{Microarray Technologies}

Computational Methods for Sequence Mapping of Large Combinatorial Libraries and Deduced Sequence Signatures) in this issue of BioTechniques.

\section{Reproducibility of R2 Indexes and Sensitivity to Probe Concentration Ratios}

The effect of probe concentration ratios on sequence assignment was evaluated by comparing the hybridization patterns between two slides, one hybridized with a $1: 2$ ratio of $C Y P 1 A 1$ and $C Y P 1 A 2$ probes (probe set 2) and the other with a 2:1 molar ratio. The R2 discrimination indexes were then compared (Figure 2C). Points clustered in the first and third quadrants with very few exceptions (less than $2 \%$ ), indicating that values of the $\mathrm{R} 2$ indexes, and thus the parental type assignments, were clear-cut, reproducible, and independent of the ratios of the probe concentrations within the range tested. Moreover, the distribution of values was centered on the coordinates $[1 ; 1]$ and $[-1 ;-1]$, indicating that the $\mathrm{R} 2$ indexes were statistically unchanged by shifts in probe concentrations. We conclude that sequence assignment is reliable and rather tolerant to fluctuations in experimental conditions.

\section{Application of Competitive Hybridization of Oligonucleotide Probes to the Analysis of Combinatorial Libraries}

Sequence attributions based on the $\mathrm{R} 2$ indexes of clones in the three spotted libraries were performed, and the data from the different probe sets were compiled. Figure 3A represents the proportion of each parental sequence at each of the six probed positions for library A. The proportion was found to be quite homogeneous for all of the sequence segments analyzed. This observation is consistent with the use of identical parental representations for all sequence segments during the PCRbased reassembly used in library constructions. However, the fluctuation of the observed proportion of CYP1A2 $(0.48 \pm 0.06)$ exceeds the expected statistical errors resulting from random sampling (about 5\% for $n=384$ ), suggesting some bias in the library. Inter- estingly, the average proportion of the CYP1A2 sequence for probed segments 2,3 , and 4 was found to be almost identical. This observation is consistent with the calculation of high $(0.95$ and 0.88 ) cross-correlation coefficients for the probability of finding an identical (either CYP1A1 or $C Y P 1 A 2$ ) parental sequence type on probed segment 2 and 3 or 3 and 4 of individual chimeras (top of Figure 3A). A correlation coefficient close to 1 indicates the near absence of

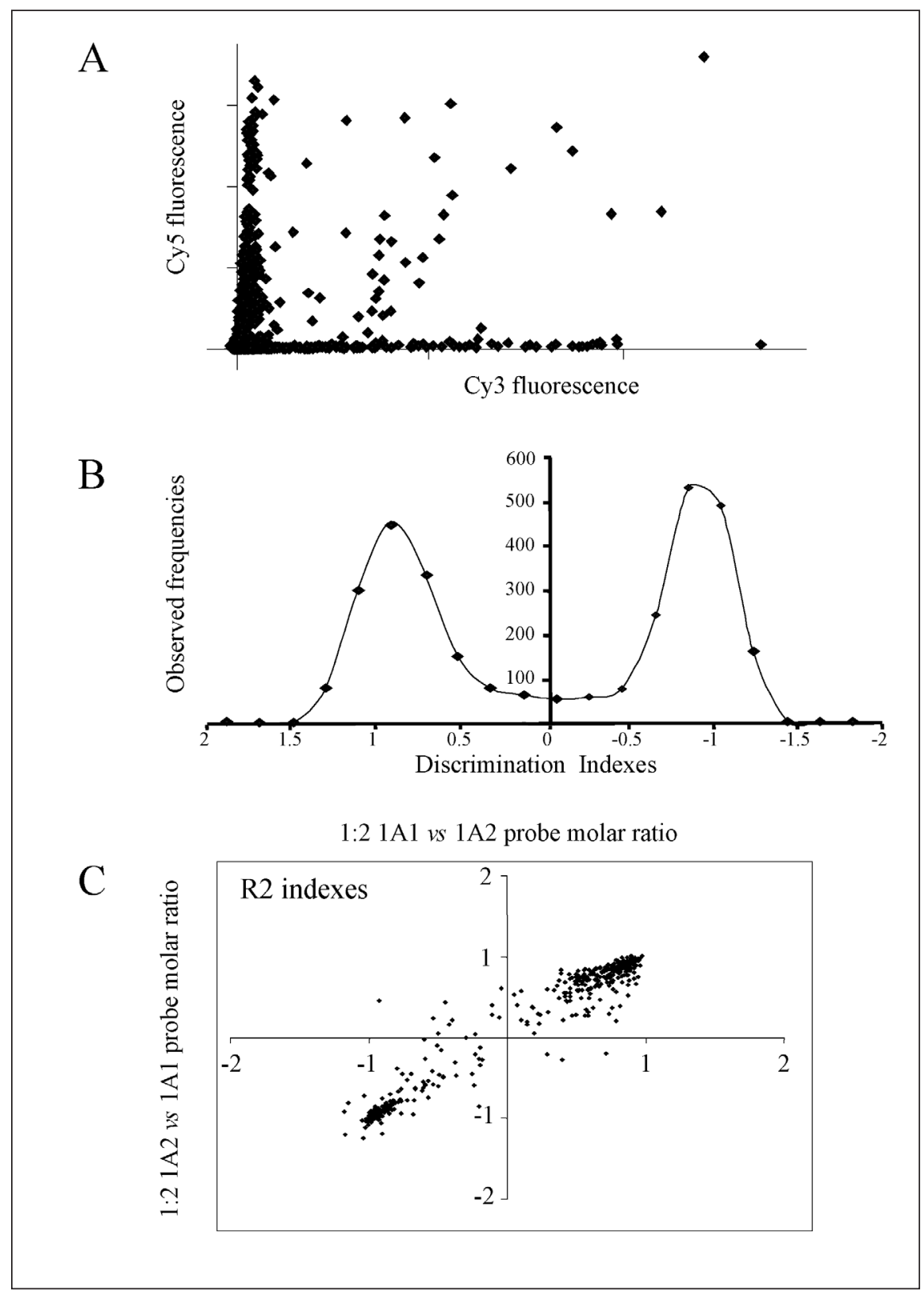

Figure 2. The relationship between $\mathrm{Cy} 3$ and $\mathrm{Cy} 5$ oligonucleotide probe fluorescence signals. (A) Cy3 intensities plotted as a function of the corresponding Cy5 intensities for all spots on the slide. (B) Frequency curve for the distribution of R2 discrimination indexes. The R2 index was calculated for each spot in a typical experiment $\left(1 \mathrm{pmol}\right.$ each probe, hybridization at $42^{\circ} \mathrm{C}$ for $1.5 \mathrm{~h}$, washing step at $35^{\circ} \mathrm{C}$ ). The experimental frequency curve for index values was calculated using a 0.2 -unit window. (C) Effect of probe concentration on R2 discrimination indexes. Two slides from the same spotting batch were used to perform hybridizations using either a $1: 2$ or a $2: 1$ molar ratio $(0.7$ and $1.4 \mathrm{pmol})$ of the Cy3-1A1- and Cy5-1A2-labeled probes (set 2 in Table 1). The $\mathrm{R} 2$ discrimination indexes relative to the 1:2 and 2:1 molar ratios are given on the horizontal and vertical axis, respectively, for the population of spots giving interpretable signals. 
recombination event between the pair of probe positions being considered, whereas a low correlation coefficient indicates near saturation by crossover events in the sequence segment between the probes. Cross-correlation coefficients were also calculated for the dataset from a previous paper (20). Similar cross-correlation coefficient values were observed for the two datasets, except in the central region where, in the microarray analysis, the cross-correlation coefficients were found to be more homogeneous among probes 2, 3, and 4 (data not shown). This seems to indicate that the dataset obtained from the microarray analysis is better, even if conclusions from both datasets are equivalent (presence of strong cross-correlation coefficients in the central region of the genes).

A much lower correlation coefficient was observed for pairs 1-2, 4-5, and 5-6 than for pairs 2-3 and 3-4, which is consistent with the presence of higher sequence similarity between the parental sequences in the sequence region between the pairs of probed sequences of the first group (Figure 3B). Similarly, the observation that the correlation coefficient for pair 5-6 was distinctly higher than the one for pairs 4-5 and 1-2 is consistent with the fact that the distance on the sequence between probes in pairs 1-

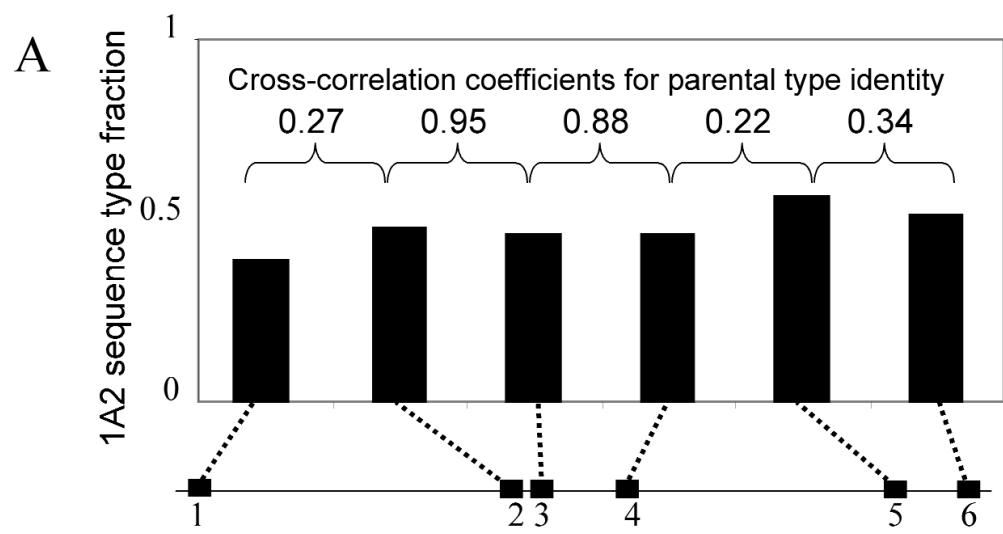

$\mathrm{B}$

Probed sequence segments (up) and positions of mismatched base pair between parental cDNA sequences (down).
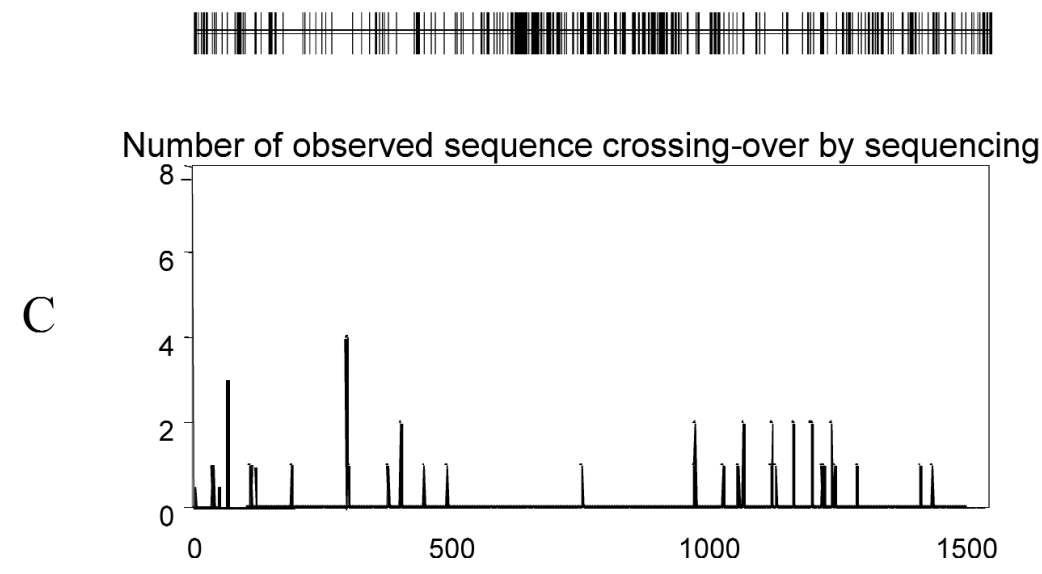

Figure 3. Correlation between microarrays and sequencing results for the distribution of crossovers. (A) Mean proportion of CYP1A2 sequence quantified at the different probe set positions in library A. Cross-correlation coefficients for the observations of an identical sequence type (either 1A1 or 1A2) on two adjacent probed sequence segments of the same mosaic gene in the library are also indicated. (B) Positions of nucleotide mismatches (vertical bars) on CYP1A1-CYP1A2 ORF alignments. The scale and alignment are the same as for panel A. (C) Observed frequency of crossover point (change of parental sequence type) following sequencing of 13 randomly chosen mosaic sequences in the library. 


\section{Microarray Technologies}

2 and 4-5 is much larger than between probes in pair 5-6, thus favoring recombination events. These findings were confirmed by ab initio calculations of crossover probabilities between CYP1A1 and CYP1A2 based on a model of PCR-mediated DNA shuffling (data not shown). The observed distribution of recombination points following full sequencing of 13 mosaic structures from the same library completely confirmed these findings (Figure 3C). Furthermore, sequencing of true "yellow" clones revealed either a mutation in the DNA at the probe position or a mixture of several plasmids. In the entire set of sequences obtained, no aberrant or nonsense sequences were found. This indicates that the DNA chip-based sequence mapping allowed access to detailed information concerning library structures and that our results can be fully confirmed by two other independent approaches. Obviously, sequence maps of individual clones that were not further analyzed in this paper constitute very useful information for library improvement, for example by robotic sorting, and for structure-function analysis.

\section{DISCUSSION}

Short probe hybridization can only be performed at low temperatures under conditions that are nondenaturing for long dsDNA. Thus, the probe can interact only with the fraction of the ssDNA already present on the slide. This fraction may be highly dependent on sample history (i.e., concentrations, residual salts, trace of detergent, and slide processing), which leads to variable hybridization signals difficult to interpret with single-labeling approaches. The use of competitive hybridization between differentially labeled (Cy3 and Cy5) oligonucleotide probes targeting overlapping sequences was found to be a significant improvement over one-probe-at-a-time strategies. The probes of a given set target overlapping sequences and thus have their hybridization similarly affected by sample quality or processing because they monitor a similar microenvironment on the chip. Notice that under the experimental conditions used, the saturation of the spots by the probes is reached after a short time so that a true kinetically controlled competition occurs. This contrasts with the situation in transcriptome analyses, in which limiting probe concentrations and long hybridization times lead to thermodynamic equilibrium. Competition between probes improves sequence discrimination and reduces, as illustrated, the sensitivity of the signal to fluctuations in experimental conditions. The intensity ratio between probes in a pair easily reaches 10-100, thus enabling clear-cut sequence discrimination. Indeed, the discrimination was found to be extremely robust to changes in probe concentrations, hybridization and washing temperatures, and hybridization times. Moreover, the results have shown that large changes in the probe concentration ratios did not result in sequence identification errors. We are currently investigating how the number of mismatches at one site can affect the consistency of the data.

The data processing we developed involves the transformation of fluorescence signal intensities in polar coordinates. The R2 index-based calculation displays good linearity of the transfer function both for low and high values of $\mathrm{Cy} 3$ and $\mathrm{Cy} 5$ relative intensities and is fairly insensitive to offset error resulting from variable backgrounds on the slide. Sequence assignment was found to be quite insensitive to experimental conditions and therefore reliable for sequence mapping of the combinatorial libraries tested.

\section{CONCLUSION}

Such a sequence map is useful to assess library quality. Moreover, in the frequent case of important bias in combinatorial libraries, it should help in the equalization and reorganization of the 
different clones by robotic sorting. Compression, by 10 - to 50 -fold, of library sizes without loss of sequence diversity can be expected, thus greatly facilitating subsequent functional screening. Analysis of changes in combinatorial library sequence maps following application of functional selection pressures might also constitute a promising approach for structure-function analysis. In conclusion, chip-based sequence mapping simplifies and improves the search for low-frequency events in molecular evolution strategies and opens new approaches to structurefunction analysis at a population-wide level in combinatorial libraries.

\section{ACKNOWLEDGMENTS}

We wish to thank Philippe Urban for the careful proofreading of the manuscript. The work was supported by a grant from CNRS for DNA microarrays and for core equipment by the GifOrsay DNA microarray platform. V.A. is a predoctoral fellow supported by a fellowship from the Ligue Nationale Contre le Cancer.

\section{REFERENCES}

1.Strezoska, Z., T. Paunesku, D. Radosavljevic, I. Labat, R. Drmanac, and R. Crkvenjakov. 1991. DNA sequencing by hybridization: 100 bases read by a non-gel-based method. Proc. Natl. Acad. Sci. USA 88:10089-10093.

2.Drmanac, R., S. Drmanac, Z. Strezoska, T. Paunesku, I. Labat, M. Zeremski, J. Snoddy, W.K. Funkhouser, et al. 1993. DNA sequence determination by hybridization: a strategy for efficient large-scale sequencing. Science 260:1649-1652.

3.Bains, W. 1993. Mixed hybridization and conventional strategies for DNA sequencing. Genet. Anal. Tech. Appl. 10:84-94.

4.Craig, A.G., D. Nizetic, J.D. Hoheisel, G. Zehetner, and H. Lehrach. 1990. Ordering of cosmid clones covering the herpes simplex virus type I (HSV-I) genome: a test case for fingerprinting by hybridisation. Nucleic Acids Res. 18:2653-2660.

5.Bains, W. 1993. Characterizing and sequencing cDNAs using oligonucleotide hybridization. DNA Seq. 4:143-150.

6.Drmanac, S. and R. Drmanac. 1994. Processing of cDNA and genomic kilobase-size clones for massive screening, mapping and sequencing by hybridization. BioTechniques 17:328-336.

7.Meier-Ewert, S., J. Lange, H. Gerst, R. Her- wig, A. Schmitt, J. Freund, T. Elge, R Mott, et al. 1998. Comparative gene expression profiling by oligonucleotide fingerprinting. Nucleic Acids Res. 26:2216-2223.

8.Drmanac, S., D. Kita, I. Labat, B. Hauser, C. Schmidt, J.D. Burczak, and R. Drmanac. 1998. Accurate sequencing by hybridization for DNA diagnostics and individual genomics. Nat. Biotechnol. 16:54-58

9.Hacia, J.G., L.C. Brody, M.S. Chee, S.P. Fodor, and F.S. Collins. 1996. Detection of heterozygous mutations in BRCA1 using high density oligonucleotide arrays and two-colour fluorescence analysis. Nat. Genet. 14:441 447.

10.Wikman, F.P., M.L. Lu, T. Thykjaer, S.H. Olesen, L.D. Andersen, C. Cordon-Cardo and T.F. Orntoft. 2000. Evaluation of the performance of a p53 sequencing microarray chip using 140 previously sequenced bladder tumor samples. Clin. Chem. 46:1555-1561.

11.Chetverin, A.B. and F.R. Kramer. 1993. Sequencing of pools of nucleic acids on oligonucleotide arrays. Biosystems 30:215-231.

12.Lipshutz, R.J., S.P. Fodor, T.R. Gingeras, and D.J. Lockhart. 1999. High density synthetic oligonucleotide arrays. Nat. Genet 21:20-24

13.Klasen, E.C., M.H. Hofker, H.M. van Paassen, M. Verlaan-de Vries, J.L. Bos, and R.R. Frants. 1987. Detection of alpha-1-antitrypsin deficiency variants by synthetic oligonucleotide hybridization. Clin. Chim. Acta 170:201-207.

14.Kurtzman, A.L., S. Govindarajan, K. Vahle, J.T. Jones, V. Heinrichs, and P.A. Patten. 2001. Advances in directed protein evolution by recursive genetic recombination: applications to therapeutic proteins. Curr Opin. Biotechnol. 12:361-370.

15.Arnold, F.H. and A.A. Volkov. 1999. Directed evolution of biocatalysts. Curr. Opin Chem. Biol. 3:54-59.

16.Cohen, N., S. Abramov, Y. Dror, and A. Freeman. 2001. In vitro enzyme evolution: the screening challenge of isolating the one in a million. Trends Biotechnol. 19:507-510.

17.Stemmer, W.P. 1994. DNA shuffling by random fragmentation and reassembly: in vitro recombination for molecular evolution. Proc. Natl. Acad. Sci. USA 91:10747-10751.

18.Crameri, A., S.A. Raillard, E. Bermudez, and W.P. Stemmer. 1998. DNA shuffling of a family of genes from diverse species accelerates directed evolution. Nature 391:288-291.

19.Joern, J.M., P. Meinhold, and F.H. Arnold. 2002. Analysis of shuffled gene libraries. J. Mol. Biol. 316:643-656.

20.Abécassis, V., D. Pompon, and G. Truan. 2000. High efficiency family shuffling based on multi-step PCR and in vivo DNA recombination in yeast: statistical and functional analysis of a combinatorial library between human cytochrome P450 1A1 and 1A2. Nucleic Acids Res. 28:E88.

21.Gibbs, M.D., K.M. Nevalainen, and P.L. Bergquist. 2001. Degenerate oligonucleotide gene shuffling (DOGS): a method for enhancing the frequency of recombination with family shuffling. Gene 271:13-20.

22.Kikuchi, M., K. Ohnishi, and S. Harayama. 2000. An effective family shuffling method using single-stranded DNA. Gene 243:133137.

23.Eickhoff, H., J. Schuchhardt, I. Ivanov, S. Meier-Ewert, J. O'Brien, A. Malik, N. Tandon, E.W. Wolski, et al. 2000. Tissue gene expression analysis using arrayed normalized cDNA libraries. Genome Res. 10:1230-1240.

24.Radelof, U., S. Hennig, P. Seranski, M. Steinfath, J. Ramser, R. Reinhardt, A. Poustka, F. Francis, and H. Lehrach. 1998. Preselection of shotgun clones by oligonucleotide fingerprinting: an efficient and high throughput strategy to reduce redundancy in largescale sequencing projects. Nucleic Acids Res. 26:5358-5364.

25.Southern, E., K. Mir, and M. Shchepinov. 1999. Molecular interactions on microarrays Nat. Genet. 21:5-9.

26.Cheung, V.G., M. Morley, F. Aguilar, A. Massimi, R. Kucherlapati, and G. Childs. 1999. Making and reading microarrays. Nat. Genet. 21:15-19

27.Duggan, D.J., M. Bittner, Y. Chen, P. Meltzer, and J.M. Trent. 1999. Expression profiling using cDNA microarrays. Nat. Genet. 21:10-14.

28.Lander, E.S. 1999. Array of hope. Nat. Genet. 21:3-4

29.Lazowski, K.W. and L. Kaczmarek. 2000. Highly sensitive detection of hybridization of oligonucleotides to specific sequences of nucleic acids by application of fluorescence resonance energy transfer. Antisense Nucleic Acid Drug Dev. 10:97-103.

30.Zhong, G.Y., J. Riov, R. Goren, and D. Holland. 2000. Competitive hybridization: theory and application in isolation and quantification of differentially regulated genes. Anal. Biochem. 282:129-135.

31.Urban, P., C. Cullin, and D. Pompon. 1990 Maximizing the expression of mammalian cytochrome P-450 monooxygenase activities in yeast cells. Biochimie 72:463-472.

32.Gautier, J.C., P. Urban, P. Beaune, and D. Pompon. 1993. Engineered yeast cells as model to study coupling between human xenobiotic metabolizing enzymes. Simulation of the two first steps of benzo[a]pyrene activation. Eur. J. Biochem. 211:63-72.

33.Diehl, F., S. Grahlmann, M. Beier, and J.D. Hoheisel. 2001. Manufacturing DNA microarrays of high spot homogeneity and reduced background signal. Nucleic Acids Res. 29:E38

34.Maniatis, T., E.F. Fritsch, and J. Sambrook. 1989. Molecular Cloning: A Laboratory Manual. CSH Laboratory Press, Cold Spring Harbor, NY.

Received 3 December 2002; accepted 20 February 2003.

Address correspondence to:

Dr. Denis Pompon

Centre de Génétique Moléculaire

Centre National de la Recherche Scientifique

UPR 2167

91190 Gif-sur-Yvette, France

e-mail:pompon@cgm.cnrs-gif.fr 